\title{
RISKS TO THE NATIONAL SECURITY GENERATED BY THE WIDESPREAD USE OF CRYPTOCURRENCY
}

\author{
George-Daniel BOBRIC \\ dbobric08@gmail.com \\ “CAROL I” NATIONAL DEFENSE UNIVERSITY, BUCHAREST, ROMANIA
}

\begin{abstract}
The pronounced multi-domain technologicalization specific to the last decades has had a significant impact on all areas of activity, including the financial one. The use of cyberspace to facilitate the actions undertaken in the monetary activity has generated the development of this field to the point where virtual currencies have been created and new technologies have been developed to support their use. Like any emerging domain, the cryptocurrency field and the related technology are in a relatively early stage and exclusively imply operating in cyberspace, thus generating security risks in the event of the involvement of malicious entities in illicit activities. In this context, it is worth analyzing how the improper use of the crypto domain can lead to various risks to national security.
\end{abstract}

\section{KEYWORDS:}

Bitcoin, blockchain technology, crypto mining, cyberspace, cyber attack

\section{Introduction}

The use of cyberspace in the current period is a ubiquitous aspect in the life of every person, either to ensure basic needs for the individual (such as socializing, relaxation, etc.), or to facilitate redundant, time-consuming activities, or even to perform complex operations. Although the benefits of using cyberspace and the resources it provides are undeniable, there is a growing trend of performing illicit actions by individuals or non-state groups with malicious intentions, stemming from the desire to fulfill a wide range of personal/collective reasons.

In this sense, the financial field and the activities performed within it or the related ones were, especially in the second half of the previous decade, the main target of the malicious actions executed in the virtual space (Fireeye, 2020), causing significant financial damage. Another important feature of the financial field specific to the last decade is the emergence on the market and the widespread use of virtual currencies, also known as cryptocurrencies. If at the beginning of the previous decade it was difficult to predict the abrupt evolution of this phenomenon, the beginning of the current decade is marked by the widespread use of these forms of virtual money transaction, as evidenced by the significant increase in the market and the increasing of the number of people using them. In this context, correlating the threats specific to the cyber 
environment with the tendency to target the malicious actions towards the financial domain and with the phenomenon of widespread use of cryptocurrencies, it can be seen that this technology is a specific vulnerability of all organizations using this form of trading; thus, by exploiting this vulnerability by individuals or non-state groups, it can become a threat to the national security. Therefore, the objective of this scientific approach is to analyze the possible problematic aspects for national security generated by the use of virtual currencies, starting from the hypothesis that, in some specific cases, the cryptocurrency can generate unwanted, significant risks to the cyber and to the financial security and, furthermore, to the national security of any state.

\section{Literature review}

This topic has been strenuously debated in recent years (mainly as an effect of the increasing level of use of these forms of trading by individuals worldwide) both in scientific, academic papers and in various groups formed by specialists in this domain of activity.

Fauzi, Paiman, and Othman (2020) analyzed the main challenges posed by the use of cryptocurrencies in everyday activities and the opportunities that this field provides to users. Among the mentioned opportunities are: the high level of security generated by the technology at the center of this field - the blockchain technology, low transaction costs, uninterrupted availability of funds, respectively high possibilities for investing in an emerging market. Frebowitz (2018) presented the main technologies related to this complex phenomenon and the vulnerabilities related to these technologies, the reasons why states choose to ban the use of virtual currency, advantages and limitations subsequent to regulatory actions of these aspects, and, last but not least, concrete examples of states that adopted or are about to adopt various legislative packages in order to project the use of cryptocurrencies outside the area of illicit activities.

In the Romanian specialty literature, a reference study for the central subject of this scientific approach entitled Current issues regarding the regulation of distributed registers technology and virtual currencies in the legislation of the Member States of the European Union (Banu \& Clem, 2019) highlights the main evolutionary lines in the field characteristic to the countries member of the European bloc and the issues of interest related to the virtual currencies. Thus, the current state of cryptocurrency regulations at the EU level as well as in the case of each member state is analyzed, showing the increased interest of the authorities to legalize different aspects related to the crypto domain.

\section{Scientific research methodology}

Both quantitative and qualitative methods have been used in this paper, delimited in methods of collecting, processing and analyzing the available information. From a qualitative point of view, the method of bibliographic documentation of the main primary and secondary sources relevant to the subject was mainly used, and on the other hand, the method of quantitative analysis was used to determine the evolution of the level of use of virtual currencies in the past years. Also, a mix between other methods and analytic tools was used in order to demonstrate the hypothesis and to fulfill the objective of the present paper.

\section{Analysis of how the population relates to virtual currencies}

During the last two years, there has been noticed greater attention of the main state and international bodies with responsibilities in regulating the financial field towards these virtual trading methods. This interest of these bodies has reflected a growing trend of cryptocurrencies' use by various users, from routine, daily activities, 
to making important transactions online. Since 2008 (the year of the beginning of the virtual currency phenomenon), various virtual currencies with different countries of origin have appeared on the cyber environment-specific trading market, which have contributed to the widespread distribution of this phenomenon and the registration of significant cryptocurrency volumes. Currently, on one of the most important profile sites, coinmarketcap.com, there are over 5,000 virtual currencies listed, which represents a real diversification of this domain.

As for the interest of users in these virtual currencies, this can be reflected through two variables: the volume (in this case, the number of cryptocurrencies traded over a period of time) and the market capitalization (representing the total value of a particular company). Thus, to analyze how users' interest in this virtual product has evolved, the data provided by the aforementioned site for the top ten most commonly used cryptocurrencies will be studied: Bitcoin, Ethereum, Tether, Binance
Coin, Cardano, Dogecoin, XRP, USD Coin, Polkadot and Uniswap (Figure no. 1). Thus, the data used to compute and analyze the trend is since 2015, with a pace of half a year, until the present, due to the fact that the cryptocurrency market was, in the first half of the previous decade, overwhelmingly composed of Bitcoin.

Figure no. 1 shows that in the last two years, there has been a high interest of the users for virtual currencies, which is reflected by the significant volume of transactions specific to the first three listed cryptocurrencies - Bitcoin, Ethereum, and Tether. In the same context, there is a growing interest in other virtual currencies, starting with the 2020 year (this is also reflected in Figure no 2). In order to be able to analyze the users' interest in the other cryptocurrencies, in Figure no. 2 the values for the volume of the three most dominant cryptocurrencies on the specific market were eliminated, so that the values can be easily observed (the raw data for plotting both figures are from the coinmarketcap.com site).

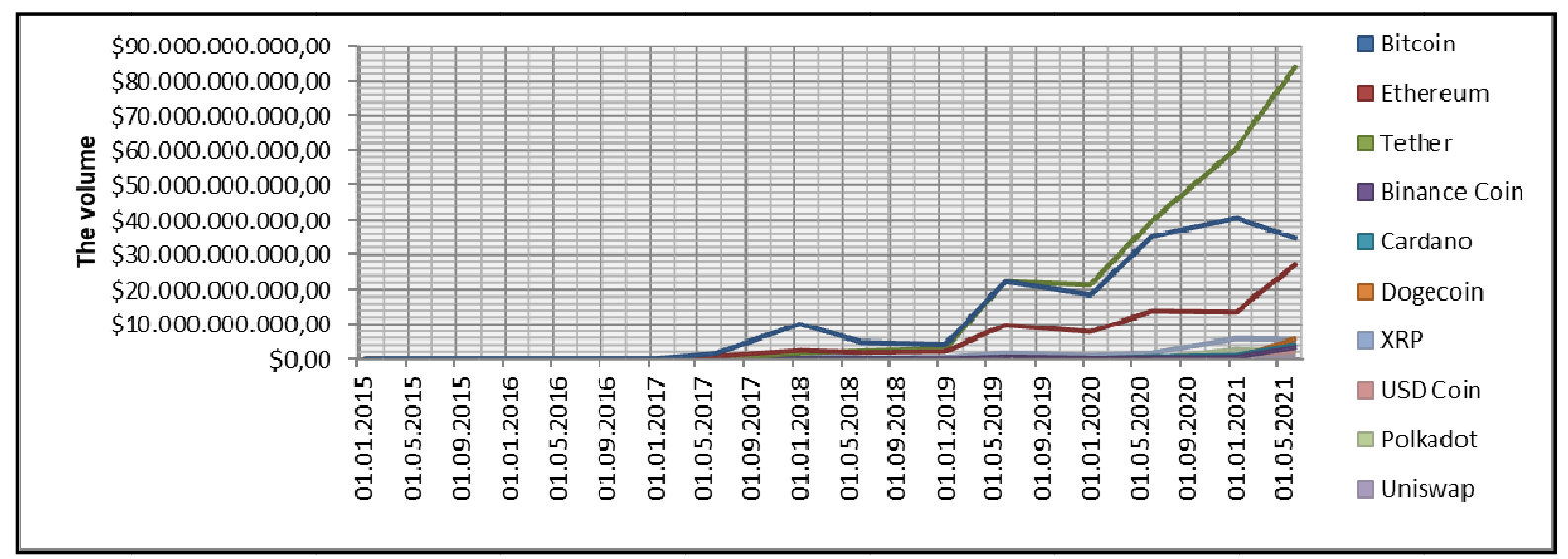

Figure no. 1: The volume of the first 10 cryptocurrencies in the 2015 - present period

Thus, Figure no. 2 graphically shows the growing trend of the population's interest in cryptocurrencies, a trend that seems to have picked up since mid-2020, probably as a result of global restrictions imposed by the coronavirus pandemic, which led to a limited ability to use physical money and increased savings for the majority of the population, which identified an opportunity to invest the savings. 


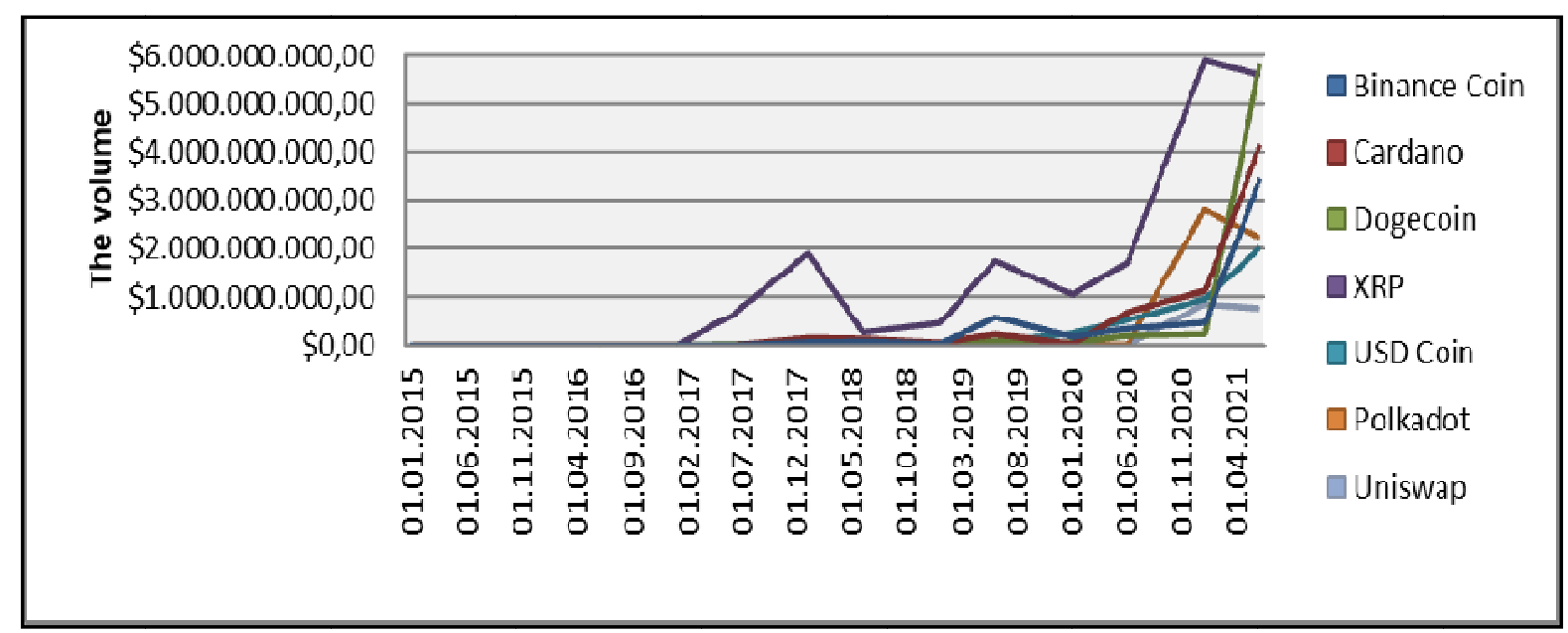

Figure no. 2: The volume of the cryptocurrencies listed on the 4-10 places in the 2015 - present period

Concluding the above-presented aspects, it can be seen that the field of cryptocurrencies has been of great interest from users especially in recent years, and, most likely, the level of their use will be increasing in the coming years.

\section{Analysis of how the authorities relate to virtual currencies}

The intention of the global population to use these forms of trading has been observed by various institutions with responsibilities in regulating the financial domain, but also by those whose field of activity is related to cybersecurity, narrowly, and to the national security, at the macro level.

At the European Union level, the need to regulate virtual currencies has been unanimously accepted, at least for the purpose of charging the potentially related incomes, but so far no accepted definition has been identified at the member state level and no common legislative framework has been created on the existence and use of these forms of trading. Currently, at the European Union level, a normative ensemble has been created meant to solve the issues related to the prevention of terrorist financing and activities related to the organized crime through virtual currency
(Cvetkova, 2018). However, there are various regulatory frameworks in place within the member states that have come into force or are in the process of being adopted, either to approve (partially or fully) the use of cryptocurrencies or to charge any potential profit from virtual currency transactions. Reaching a compromise at the European level is difficult mainly due to the fact that the technological development of this field is taking place at a much faster rate than the completion of the regulatory process, to which is added the emergence of an overwhelming number of new virtual currencies that are flooding the market corroborated with the users' growing appetite for cryptocurrencies and the potential gains they provide.

In the same context, in order to analyze the interest of the competent authorities in the field of cryptocurrencies, it is interesting to study how they were regulated by the three world superpowers: the United States, the People's Republic of China, and the Russian Federation. In the case of the first one, the same situation persists as in the case of the European Union, there being various legislative packages at the level of each component state entity, but there is no consensus in this regard at the federal level. Overall, in the US, the attitude towards the 
use of cryptocurrencies is a positive one; until 2019 more than half of the member states had adopted various legislative frameworks for the use of cryptocurrencies and the related technology. This is also aided by the fact that the federal government has not made use of the constitutional power to regulate this area, leaving these decisions in the power of the responsible state bodies at the level of each state (Business Insider, 2021).

On the other hand, the case of the People's Republic of China is a completely different approach from that of the United States, with the Chinese authorities having a repugnant attitude towards the use of cryptocurrencies and their related technology. In connection with the field of virtual currency, the Chinese authorities have (Goitom, 2018):

- prohibited the collection of funds through the process of initial coins offering, activity specific to the cryptocurrencies domain and its related technology;

- imposed significant restrictions on cryptocurrency trading platforms;

- applied specific requirements to financial-banking institutions not to support the activities in the spectrum of the virtual currency transactions;

- carried out various actions to discourage Bitcoin mining.

As for the Russian Federation, the beginning of 2021 was a starting point in the process of regulating the cryptocurrencies domain, by adopting a law to address various issues regarding the issuance, accounting and use of virtual financial assets. In this sense, Russian specialists (Shovkhalov \& Idrisov, 2021) appreciate that the level of consolidation of the Russian regulatory framework on cryptocurrencies is not enough, possible developments in the legislative spectrum may arise simultaneously with the development of the virtual currency domain.

In conclusion, as in the case of ordinary consumers, there is an increased interest of the authorities in the cryptocurrencies domain. In the next subchapter some of the aspects that generate this interest from the institutions with competencies in the financial spectrum, respectively from the entities from organic state apparatuses with attributions regarding financial, cyber and/or national security, will be analyzed.

\section{Risks related to the misuse of cryptocurrencies and their related technologies}

Cyberspace is characterized by the existence of numerous opportunities, generated by its peculiarities, through which vast operations and complex actions can be carried out. These characteristics are also the ones that determine the existence of numerous vulnerabilities that, in the context of their opportune exploitation by people with malicious intentions, can materialize in more and more varied threats. In the same context, the excessive technological evolution specific to the last years has determined an important increase of the level of technologicalization of the fields of activity, this being also the case of the financial domain.

Various specialized studies have been developed by researchers on the risks arising from the use of cyberspace for illicit purposes. For example, Mittal (2017) presents the risks to be grouped on three levels: addressing the physical components that make up the systems that support the cyber ecosystem; on possible services through cyberspace; to the information content stored or in transit.

Having its debut period in 2008, the cryptocurrencies domain registered a constant evolution in the first years, the market being formed only by Bitcoin and, subsequently, new cryptocurrencies were established that threatened/threatens the hegemony of the first virtual currency. Simultaneously with the evolution of this field of activity, new technologies have been developed to facilitate operations with this type of virtual assets. 
Combining the risks posed by the emergence of this area with the illicit actions performed in the fifth operational environment - the cyber domain, it can be seen that there is a wide range of threats to cybersecurity and, at the macro level, to national security.

These risks could be analyzed from a multitude of different angles but, for the purpose of this paper, they will be divided into two main categories: related to the technology at the core of the cryptocurrency trading process, respectively risks generated by the volatility and instability of these virtual currencies. In this sense, in the current scientific paper, only the main risks related to the technological parts of the cryptocurrency field will be analyzed.

At the basis of the existence of these forms of virtual trading is the blockchain technology. An equivalent, in the real world, of this technology could be the registers used to record financial transactions. In the same (simplistic) idea, blockchain technology allows the "storage" of a large number of transactions not only financial, but also with other valuables. In fact, when two users intend to initiate a cryptocurrency exchange, they actually initiate a transaction through a public key and a private key. At this moment, the system verifies, through the blockchain technology, whether the initiator of the transaction has the amount of cryptocurrencies for which he initiated the transaction by searching in the "virtual registers"; if so, the transaction is accepted and a new link called "block" is added to the "chain"; in a negative sense, the transaction is rejected (Goel et al., 2018).

Being a field whose core technology is found entirely in the cyber environment, the main risks are generated by its illicit use mainly to obtain financial gains by conducting cyberattacks of various types. The most common such attack is the phishing attack, its main purpose being the identification, by the attacker, of the victim's credentials, in order to be able to use them later in order to request sums of money as ransom. One such case (but not the only one) is the one discovered by Cisco in early 2017, generically called the Coinhoarder Campaign. This campaign, located in Ukraine, was directed against the most popular cryptocurrency, Bitcoin, by targeting the main specific wallet site, blockchain.info. The innovative element, at that time, was the fact that the cyber group behind these actions was illegally using an advertising service offered by Google, called Google AdWords, used by certain companies to facilitate their promotion through the search engine. Thus, the average user accesses such a fake site and the illegal group steals the virtual wallets of the user victim (Holub \& O'Connor, 2018). Another important case is the one presented by the F-Secure company (2019) regarding the operations carried out by the Lazarus group (being part, according to the specialty literature, to the Democratic People's Republic of Korea) against a company in the cryptocurrency sector. This operation was interconnected to a phishing campaign underway (at the time) worldwide, with the group demonstrating a high level of sophistication in executing a cybercrimespecific campaign.

Another aspect of interest when analyzing the risks generated by the use of cryptocurrencies occurs as a result of a phenomenon common in recent years: the compromise, through cyber attacks, of trading platforms involved in the processes that take place between the public addresses. Virtual currencies are a good asset for cybercriminals and a way in which their intrinsic desire to get rich and, as far as possible, to escape law enforcement, can be fulfilled, so that the number of such actions reflects the trend of the significant increase in the volume of actions specific to the cyber fraud (Bobric, 2021). In this context, the attackers target these trading platforms through a variety of different methods, and if the attack is successful, the money is transferred through as many accounts as possible, usually thousands, in an attempt to 
lose the track of money. Finally, the attacker is forced to use a special service in order to exchange the virtual in physical currency. Following this logic of activities, after significant efforts to identify the accounts through which the cryptocurrencies were passed starting from the first account known to have been hacked, the law enforcement agencies can identify the final service through which the virtual currency was exchanged in the fiat forms, having a first valid indication regarding the attacking person/group. Thus, the amount of cryptocurrencies stolen through this process over a period of nine years (between 2011 and 2019) reached a level of about one billion and seven hundred million dollars the equivalent in cryptocurrencies (Goldsmith, Grauer \& Shmalo, 2020).

On top of the most important risks, infecting cryptocurrency mining stations with malware would occupy the leading places. This illegal way of acquiring virtual financial assets is used through two main directions of action:

- exfiltration of the financial amount existing at the time of the attack in the victims' virtual wallets, a situation which would be reduced to the situation presented in the previous paragraph;

- mining of cryptocurrencies through the infected devices. In a narrow sense, this activity could be explained in not very technical terms as the process of mathematical evaluation of data blocks in order to validate transactions between different users (Ankalkoti \& Santhosh, 2017). This process requires a device (computer, phone, tablet, etc.) with the highest possible processing capacity and a very good Internet connection, so that the miner can provide the mathematical solution in exchange for the financial reward in the amount of cryptocurrency parts. In this way, through malware programs, the attackers impersonate the devices to use them for their own purpose as a result of using the victim's mining resources in a hidden, illegal way.

\section{Risks to the national security}

Regarding the risks to the national security, they are multiple, some of which (those considered to be more important) are analyzed in this subchapter. The cyber domain-related risks presented above, which have a particularly significant impact on the cryptocurrency trading process, when carried out on a large scale, can significantly affect the national security, especially its financial-specific subcomponent. Closely related to this risk is the possibility of using cryptocurrencies by cybercrime groups or by lone, malicious and solid technical actors to achieve their own objectives, mostly subsequent to the desire to obtain financial assets by illicit methods. Thus, cyberspace can facilitate both obtaining funds through illegal methods, but also the operation of covert transactions, without the monetary volume to be verified by the competent authorities. For example, in order to carry out transactions with sums of money higher than a certain maximum threshold established by law, groups, organizations or individuals are required to execute these transactions through the bank, in which case it is necessary to complete declarations of origin of the money. In the case of funds obtained from illegal activities, this aspect is problematic, so that cryptocurrencies are a very effective method to launder money, to carry out transactions without attracting the attention of the authorities and, finally, to lose the trace of money. In conclusion, a risk to the national security generated by the existence of the cryptocurrencies domain is represented by the activities of cybercrime groups subscribed to money laundering and tax evasion.

In the same vein, an equally significant risk subsumed to the existence of the cryptocurrency trading platforms is generated by the use of these virtual financial formats to support the actions of terrorist groups. Through cyberspace and cryptocurrencies, terrorist actions can be facilitated as follows (Dion-Schwarz, Manheim \& Johnston, 2019): 
- funding by various donor entities, who wish to remain anonymous but identify themselves with the cause for which the terrorist organization is active;

- receiving the equivalent value for various goods supplied (for example, arms trafficking) or services provided (for example, drug transport), representing the source of income for the terrorist group;

- the transfer of money to finance activities between members of the group or from the main group to individuals in various parts of the world, in order to provide the financial support needed to carry out the tasks set;

- payment of the value of some goods and services necessary for the development in optimal conditions of the planned activities.

Another risk to national security is generated by the volatility of the equivalent prices of cryptocurrencies, relative to their value in the amount of classical currencies. This increased volatility can be the cause of a high number of speculative losses, which can create chaos in the investment market, price increases, dissatisfaction among the population, etc.

Similarly, a significant risk is related to the legislative field. As previously presented, the interest of state and international bodies to regulate, in various forms, these forms of virtual currency, is growing. Thus, the lack of a legislative package that regulates the field of cryptocurrencies at the international level, from the possibilities of creating new cryptocurrencies to the ways of trading and using them in daily activities, can lead to a series of inaccuracies that can be used by various individuals or groups for their own benefit, to circumvent state authorities and applicable laws. In this regard, questions arise as to how the audit process will be able to be carried out in the case of these currencies, in the event that significant fluctuations in the value of cryptocurrencies on the current market may generate a series of speculative attacks that create risks of economic nature of a particular sector of activity and which, on the basis of the snowball effect, may lead to financial risks to national security.

Moreover, in the same idea, KnowYour-Customer protocols are used to prevent actions subsumed to illicit money laundering activities. Following a study developed by the organization CypherTrace (2020), these types of protocols were analyzed belonging to a number of over 800 virtual asset service providers in over 80 countries, the results being presented in Figure no. 3.

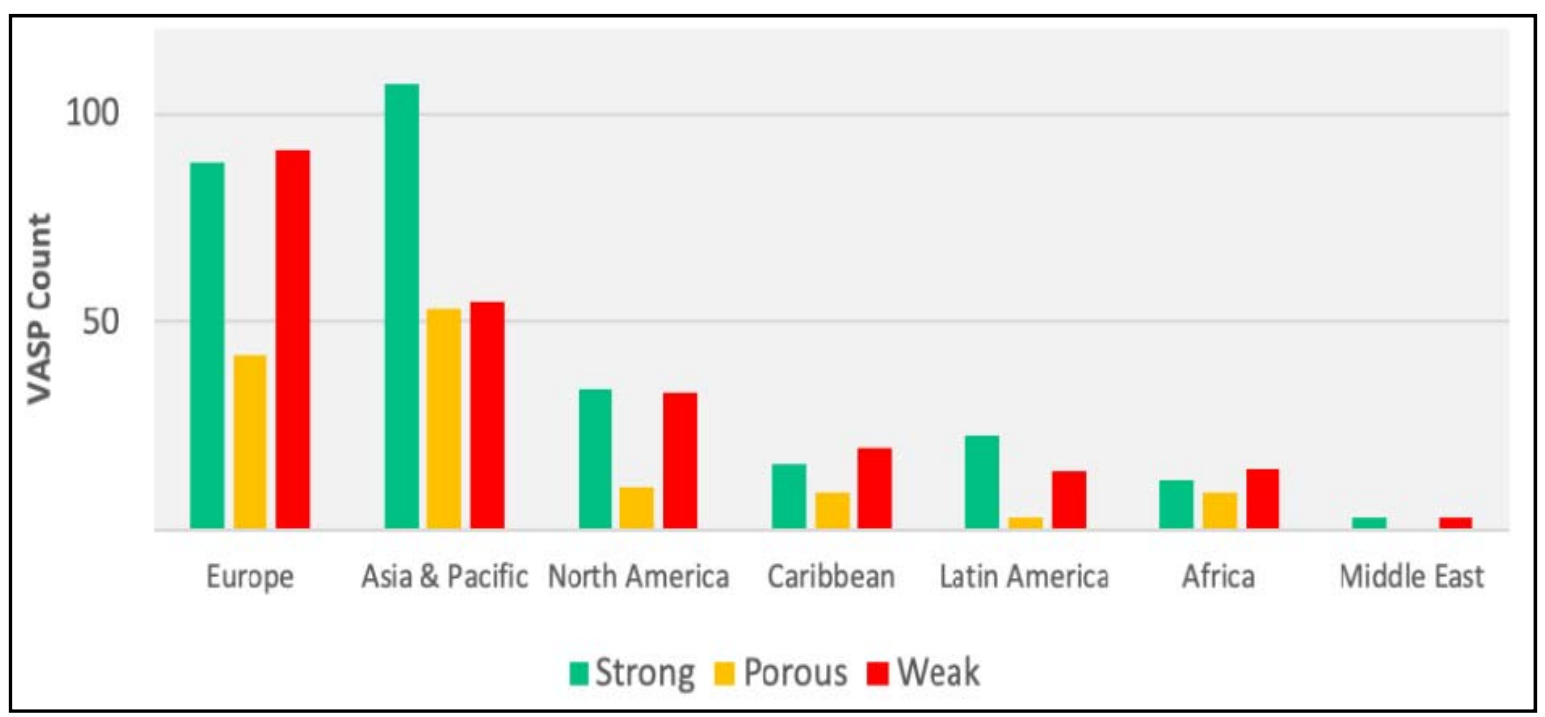

Figure no. 3: Anti-laundering protocols' complexity

(Source: Ciphertrace, 2020) 
As it can be seen from Figure no. 3, the European continent has the weakest protocols implemented at the level of virtual asset service providers, followed in this regard by Asia and North America. If we look at these facts, it turns out that most users worldwide (geographically, most cryptocurrency users are in Europe, North America and Asia) use the services of providers whose policies for the limitation of activities subject to money laundering are not implemented correctly or are not strong enough. It is estimated that, according to the data provided by the study, about a third of service providers have implemented weak protocols to prevent money laundering through cryptocurrencies, to which is added about a quarter of the total number of more permissive protocols. With this in mind, it can be seen that, at present, the risks to national security specific to the financial field can be increased by the use of cryptocurrencies in illicit actions related to the field of money laundering by various entities.

\section{Conclusions}

Undoubtedly, the cryptocurrencies domain represents the future of the financial domain; an aspect proved both by the increased interest of the population for this new form of trading, and by the consistent and numerous steps of the relevant authorities to regulate, at least nationally, various issues related to the crypto-domain. At the same time, the opportunities generated by the cryptocurrencies domain (investments, improvements of banking services, reduction of time spent in physical banks, elimination of bureaucracy, etc.) will determine an even higher number of users to make the transition to virtual currency.

As previously presented, global interest in cryptocurrencies has intensified especially since 2020 , and this can be attributed to the coronavirus pandemic. The restrictions on movement imposed worldwide have made it impossible for people to conduct banking transactions by physically being present at their headquarters, so the population has been forced to migrate entirely to the cyber environment, by conducting online transactions, where possible. At the same time, the restrictions of movement have caused significant losses to a very large number of businesses, so some investors have sought and identified a new investment opportunity: the purchase of cryptocurrencies. Corroborating these two aspects, it can be concluded that the coronavirus pandemic has led to an increased interest of the global population for cryptocurrencies, as demonstrated by the figures previously presented and analyzed. In this context, the interest in this part of the financial field is also supported by the opportunities generated by its very existence, including the reduced cost and time to carry out a transaction, the possibility to transfer cryptocurrencies from any corner of the world to any other destination in a matter of few seconds, reduction of bureaucracy to a minimum and the possibility to make payments through an application, encouraging entrepreneurs to start a new business starting with cryptocurrencies, etc.

Although the first half of the last decade was characterized by a predominantly Bitcoin market, the first cryptocurrency established, the end of the decade demonstrated an emergence of new cryptocurrencies around the world, and the fact that their value continues to grow is still a clue of the fact that the world is moving towards a future in which the role of cryptocurrencies will be recognized worldwide. In this sense, the emergence of this field and the rapid international development of new cryptocurrencies generate a number of major risks to national security, both technological (mainly in the field of cyberattacks), but also of a financial nature (especially from the spectrum of money laundering or tax evasion) or other more serious types, such as terrorist financing. Therefore, there is a major interest both at the individual state and international level, to regulate as strictly as 
possible this domain, the steps in this regard being channeled on three directions of action: total ban, partial ban/acceptance and full acceptance. At the beginning of the road, these approaches face, first of all, the impossibility of keeping up with the evolutionary trend that characterizes cryptocurrencies, this aspect being, in fact, another risk to the national security and, to a greater extent, to the regional or even international security.

In other words, as previously presented, from the point of view of financial security, the implementation of solutions to limit the risks related to this technology has not been successful so far. Surprisingly, countries where this form of finance is predominantly used allow the existence of services from providers who have not implemented appropriate anti-money laundering protocols. This is a concrete example of the fact that, in the face of this new technology, competent authorities, service providers and even consumers do not have all the necessary solutions to limit the negative effects of a potential malicious use of these trading facilities.

Regarding the cryptocurrency market in Romania, it can be appreciated that, in the near future, the majority of the population will have an intention of purchasing various amounts of this virtual asset. This fact is also due to the existence of a local cryptocurrency, called Elrond, which aroused the interest of the population for cryptocurrencies through the success it has shown in recent years. Moreover, the success of the entrepreneurs who created Elrond is a model for a significant number of capable young people who, in a country with very good Internet infrastructure, can create new solutions in the cryptocurrencies domain.

In conclusion, cryptocurrencies represent the transition to the new, to development, to the future. Instead, currently, their use by various actors with malicious intentions (individuals eager to get rich illegally, cybercrime groups, etc.), coupled with the low level of regulation of the functioning of this domain and the low degree of implementation of some policies that limit any such unwanted uses, can lead to risks to cyber and financial security and, depending on the level of threat, even to the national security.

\section{REFERENCES}

Ankalkoti, P., \& Santhosh, S.G. (2017). A Relative Study on Bitcoin Mining. Imperial Journal of Interdisciplinary Research, Vol. 3, 1757-1761, available at: https://www.researchgate. net/publication/318850089_A_Relative_Study_on_Bitcoin_Mining, accessed on July 2021.

Banu, C.M., \& Clem, F. (2019). Aspecte de actualitate privind reglementarea tehnologiei registrelor distribuite și a monedelor virtuale în legislația statelor membre ale Uniunii Europene, available at: http://www.cdep.ro/afaceri_europene/afeur/2019/st_2643.pdf, accessed on June 2021.

Bobric, G.D. (2021). The Evolution of Cyber Fraud in the Past Decade. Proceedings of the $20^{\text {th }}$ European Conference on Cyber Warfare and Security, 44-51.

Bussines Insider. (2021). How the laws \& regulations affecting blockchain technology and cryptocurrencies, like Bitcoin, can impact its adoption. Available at: https://www.businessinsider.com/blockchain-cryptocurrency-regulations-us-global, accessed on May 2021.

Ciphertrace. (2020). 2020 Geographic Risk Report: VASP KYC by Jurisdiction. Available at: https://ciphertrace.com/2020-geo-risk-report-on-vasp-kyc/, accessed on June 2021. 
Cvetkova, I. (2018). Cryptocurrencies legal regulation. BRICS Law Journal, Vol. 5, 128-153, available at: https://www.researchgate.net/publication/326195399 Cryptocurrencies legal regulation, accessed on May 2021.

Dion-Schwarz, C., Manheim, D., \& Johnston, P. (2019). Terrorist Use of Cryptocurrencies: Technical and Organizational Barriers and Future Threats. RAND Corporation, available at: https://www.researchgate.net/publication/332214878 Terrorist Use of Cryptocurrencies_Technical_and_Organizational_Barriers and Future Threats, accessed on July 2021.

F-Secure. (2020). Lazarus Group campaign targeting the cryptocurrency vertical, available at: https://labs.f-secure.com/assets/BlogFiles/f-secureLABS-tlp-white-lazarusthreat-intel-report2.pdf, accessed on June 2021.

Fauzi, M.A., Paiman, N., \& Othman, Z. (2020). Bitcoin and Cryptocurrency: Challenges, Opportunities and Future Work. Journal of Asian Finance Economics and Business, Vol. 8, 695-704, available at: https://www.researchgate.net/publication/345 003534_Cryptocurrency_Threat_or_Opportunity/link/5f9bf1fb299bf1b53e514b63/download, accessed on June 2021.

Frebowitz, R.L. (2018). Cryptocurrency and state sovereignty. Available at: https://www.semanticscholar.org/paper/Cryptocurrency-and-State-Sovereignty-Frebowitz/ 2cc0f13e948 63bab77171679699d797f467824f, accessed on May 2021.

Goel, N. et al. (2018). Security issues in Blockchain and crypto currency. Available at: https://www.researchgate.net/publication/338660128_Security_issues_in_Blockchain_and_cr ypto_currency/link/5e221 ccfa6fdcc1015717035/download, accessed on June 2021.

Goldsmith, D., Grauer K., \& Shmalo Y. (2020). Analyzing hack subnetworks in the bitcoin transaction graph. Applied Network Science, Vol. 5, 2, available at: https://www.researchgate.net/publication/340710841_Analyzing hack subnetworks in the bitcoin transaction graph, accessed on June 2021.

Holub, A., \& O'Connor, J. (2018). COINHOARDER: Tracking a Ukrainian Bitcoin Phishing Ring DNS Style. Available at: https://docs.apwg.org/ecrimeresearch/2018/ 5369253.pdf, accessed on June 2021.

Mittal, S. (2016). Risks and Opportunities Provided by the Cyber-Domain and PolicyNeeds to Address the Cyber-Defense. International Research Journal on Police Science, available at: https://papers.ssrn.com/sol3/papers.cfm?abstract $\mathrm{id}=2975895$, accessed on June 2021.

Shovkhalov, S., \& Idrisov, H. (2021). Economic and Legal Analysis of Cryptocurrency: Scientific Views from Russia and the Muslim World. Laws, Vol. 10, Issue 2, 1-17, available at: https://doi.org/10.3390/laws10020032, accessed on June 2021.

Zhang, L. (2018). Regulation of Cryptocurrency in Selected Jurisdictions, 30-34, available at: https://www.loc.gov/law/help/cryptocurrency/regulation-of-cryptocurrency.pdf, accessed on June 2021. 